\title{
PDE Boundary Control for Flexible Articulated Aircraft Wings
}

\author{
Aditya A. Paranjape*, Soon-Jo Chung $†$ and Miroslav Krstic ${ }^{\ddagger}$
}

\begin{abstract}
The paper considers a boundary control formulation for PDEs with a system output given by a spatial integral of weighted functions of the state. This formulation is directly applicable to the control of an aircraft with articulated flexible wings, in which case the output of interest is a net aerodynamic force or moment. Flexible wings can be controlled via actuation at the root or the tip. The problem of beam twist is analysed in detail to illustrate the formulation, and it shown that the control law ensures that the error between the desired output signal and the actual output signal decreases exponentially to an uniform ultimate bound. Stability of the closed loop system is proved by Lyapunov techniques. The formulation is demonstrated by simulations.
\end{abstract}

\section{Nomenclature}

$\begin{array}{cl}c & \text { wing chord length } \\ E, G & \text { elastic modulus and shear modulus of the wing material } \\ g & \text { gravitational constant } \\ I_{y y}, J & \text { second moment of area of the airfoil and torsion constant } \\ m, I_{p} & \text { mass per unit length of the wing, airfoil second moment of inertia } \\ V & \text { flight speed } \\ x_{a}, x_{e} & \text { non-dimensional distance of aerodynamic center and airfoil center of mass } \\ \delta_{R}, \theta_{R} & \text { from shear center } \\ \eta & \text { bending slope (dihedral) and incidence angle at the wing root } \\ \theta & \text { Kelvin-Voigt damping coefficient } \\ \xi & \text { bending displacemnt } \\ \text { Notation } & \text { time and spanwise spatial variable } \\ t, y & \frac{\partial v}{\partial t} \text { and } \frac{\partial v}{\partial y} \\ v_{t}, v_{y} & \frac{\partial^{3} v}{\partial t \partial y^{2}} \text { (and so on) } \\ v_{t y y} & \text { time derivative of a variable } v(t) \\ \dot{v} & \end{array}$

\section{Introduction}

A new concept for controlling micro aerial vehicles, based on wing articulation, was introduced in Refs. [1, 2]. The concept lends itself readily to aeroelastic tailoring. Aeroelastic tailoring is seen as an asset in the

\footnotetext{
* Doctoral candidate, Department of Aerospace Engineering, Univ. of Illinois at Urbana-Champaign (UIUC), Urbana, IL. Email: paranja2@illinois.edu; Student Member, AIAA.

$\dagger$ Assistant Professor, Department of Aerospace Engineering, University of Illinois at Urbana-Champaign Email: sjchung@illinois.edu; Senior Member, AIAA.

${ }^{\ddagger}$ Daniel L. Alspach Professor of Dynamic Systems and Control, Dept. of Mechanical and Aerospace Engineering, Univ. of California, San Diego, La Jolla, CA 92903. Email: krstic@ucsd.edu.
} 


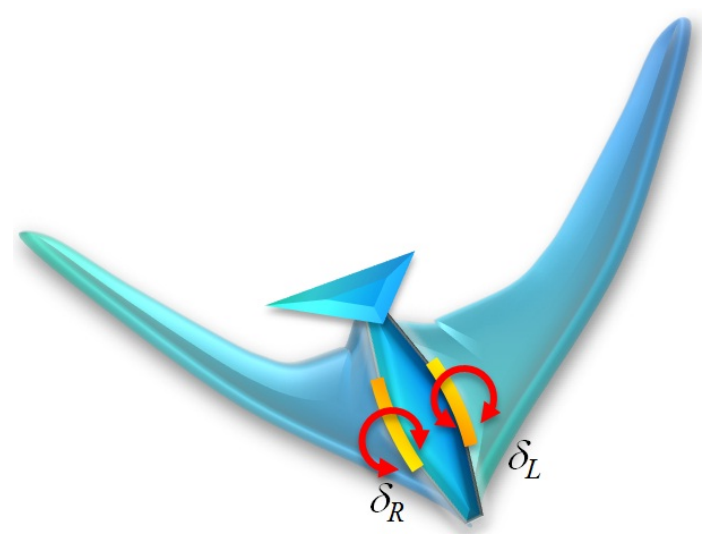

(a) Root actuation

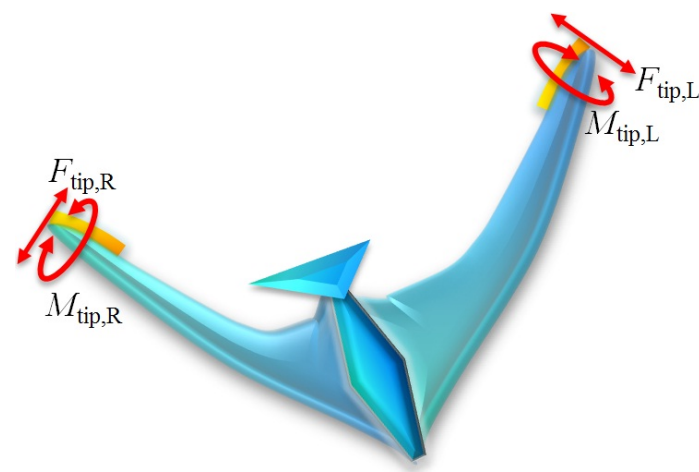

(b) Tip actuation

Figure 1. A schematic showing an MAV with highly flexible wings controlled at the wing root and wing tip, respectively.

development of agile micro aerial vehicles. ${ }^{1,3-5}$ Wing flexibility not only improves aircraft performance and stability passively, but can also be actuated actively for control. ${ }^{1,3,6}$ Figure 1 shows a conceptual MAV with highly flexible wings whose deformation can be controlled actively by actuators located at the wing root and/or the wing tip.

Broadly speaking, continuous systems such as wings can be controlled in two ways: distributed control and boundary control. The former approach relies on a series or a network of actuators and sensors distributed over the system. The latter approach relies on actuators deployed only on the boundary of the system under consideration. For practical engineering applications, the most important benefit of boundary control is the reduction in the number of actuators. Consider, for example, a flexible wing mounted on a miniature aircraft. This example is illustrative of the reverse engineering of bird flight. Whereas feathers distributed all over the wing are known to play an active role in flight control, installing such a distributed scheme would incur substantial penalties in weight as well as costs. The benefits of distributed actuation can be made up, in part, by a combination of good design and sound boundary control. This paper presents a boundary control (BC) problem for wing twist which could be extended to a wider class of hyperbolic partial differential equations (PDEs).

There is a substantial amount of literature on boundary control theory of PDEs (see Ref. [7-12] for material pertinent to this paper and the references cited therein). There are two sets of methods for boundary control of PDEs. The first approach involves converting the PDEs into ordinary differential equations using approximation methods such as those of Galerkin or Rayleigh-Ritz. ${ }^{11,13}$ This renders the problem amenable to control by any of a large number of linear and nonlinear methods for control of systems described by ODEs. ${ }^{13}$ The second approach involves keeping the PDEs intact, and using a "model-following" approach as described in a recent book by Krstic and Smyshlyaev. ${ }^{9}$ In particular, this approach seeks to achieve an $a$ priori prescribed deformation. This is indeed the objective of problems such as maneuvering robotic arms ${ }^{9,14}$ or suppressing vibrations in a flexible beam. ${ }^{15}$ On the other hand, in problems such as those involving flexible aircraft wings where the control objective would be to achieve a net aerodynamic force or moment, there could be several candidate deformations. Prescribing one particular candidate deformation places a greater demand on the control system.

It is worth noting that if a PDE is approximated by Galerkin's method, the problem of achieving an integral objective reduces to a standard output control problem. Whereas solutions to output control problems in an ODE setting are abundantly known, an ODE-based approximation to PDEs usually results in systems having large orders. It is not very easy to incorporate a time-varying boundary condition into the basis functions which results in the possibility that the control design process becomes tedious and the control laws non-intuitive. Ref. [10] also points out that a finite-state approximation may wrongly render fundamental system theoretic properties like controllability and observability to be functions of the approximation.

In this paper, the control objective is restricted to achieving the net aerodynamic force or moment, an abstraction of which is ensuring that some weighted integral of the deformation equals the prescribed value. 
A similar idea for a more general class of PDEs has been explored in Refs. $[10,11]$ in the context of spatially distributed control. This paper derives a tracking boundary controller for ensuring the that the output tracks the desired reference signal. An adaptive controller for a limited class of parametric uncertainties is also derived.

This paper is organized as follows. The problem formulation is explained in Sec. II. A backstepping-based control scheme, inspired by Ref. [9], is derived in Sec. III. A control scheme based on actuation at the wing tip and its adaptive version are presented in Sec. IV. Preliminary results for nonlinear aerodynamics are derived in Sec. V. Numerical simulations are presented in Sec. VI.

\section{Problem Formulation}

\section{A. Motivation}

The motivation for considering the problem of boundary control of beams comes from the problem of controlling flexible wings for rapid aircraft maneuvers. Suppose that the wing has length $L$, and the spanwise coordinate is denoted by $y$. Let $E I$ and $G J$ denote the bending and torsion stiffness, respectively. The wing has a linear mass density $m$, and the polar moment of area is given by $I_{p}(y) \equiv I_{p}$. The wing is loaded transversely with a load $F(\xi, \theta, \mathbf{u})$, where $\xi \triangleq \xi(t, y)$ and $\theta \triangleq \theta(t, y)$ denote the bending and twist displacements, respectively. The resultant wind velocity at a given point on the wing, $\mathbf{u}$, is a sum of the aircraft flight speed $\mathbf{u}_{B}$ (measured in the body axis) and the wing vibration rate $\mathbf{u}_{f}==\left[\begin{array}{lll}0 & \dot{\xi}\end{array}\right]^{\mathrm{T}}$, so that $\mathbf{u}=\mathbf{T}_{W B} \mathbf{u}_{B}+\mathbf{u}_{f}(y)$, where $\mathbf{T}_{W B}$ is the rotation matrix from the body frame to the local wing frame and $\mathbf{u}_{f}$. Let $c$ denote the wing width, and $x_{e} c$ denote the distance between the center of pressure and the shear center of the wing.

Then, the equations for wing vibration dynamics are given by ${ }^{1}$

$$
\left[\begin{array}{cc}
m & -m x_{e} c \\
-m x_{e} c & I_{p}
\end{array}\right]\left[\begin{array}{c}
\xi_{t t} \\
\theta_{t t}
\end{array}\right]+\left[\begin{array}{c}
\eta E I \xi_{t y y y y}+E I_{y y} \xi_{y y y y} \\
-\eta G J \theta_{t y y}-G J \theta_{y y}
\end{array}\right]=\left[\begin{array}{c}
F\left(\xi_{y}, \theta, \mathbf{u}, \dot{\mathbf{u}}_{B}, g\right) \\
-x_{a} c F\left(\xi_{y}, \theta, \mathbf{u}, \dot{\mathbf{u}}_{B}, g\right)
\end{array}\right]
$$

where $x_{a}$ and $x_{e}$ denote the normalized distances (with respect to the chord length $c$ ) of the aerodynamic center and the center of gravity of the wing from the shear center. The subscripts $t$ and $y$ denote partial derivatives, i.e., $\xi_{t t}=\frac{\partial \xi}{\partial t^{2}}, \xi_{\text {tyyyy }}=\frac{\partial \xi}{\partial t \partial y^{4}}$, and so on. This equation is valid for the right wing, and a similar equation can be derived for the left wing as well. Note that $F$ depends on $\dot{\mathbf{u}}_{B}$, which is the contribution from inertial effects arising from aircraft acceleration and the added mass effect. ${ }^{2}$ bd The boundary conditions for tip based actuation are given by

$$
\begin{aligned}
& \xi(0)=0, \quad \xi_{y}(0)=0, \quad \xi_{y y}(L)=0, \quad \xi_{y y y}(L)=\frac{F_{\mathrm{tip}}}{E I_{y y}}, \\
& \theta(0)=0, \quad \theta_{y}(L)=\frac{M_{\mathrm{tip}}}{G J}
\end{aligned}
$$

while those for root-based actuation are

$$
\begin{aligned}
& \xi(0)=0, \quad \xi_{y}(0)=\delta_{R}, \quad \xi_{y y}(L)=0=\xi_{y y y}(L)=0, \\
& \theta(0)=\theta_{R}, \quad \theta_{y}(L)=0
\end{aligned}
$$

In Eqs. (2) and (3), $F_{\text {tip }}$ and $M_{\text {tip }}$ are the applied tip force and twisting moment, respectively, while $\delta_{R}$ and $\theta_{R}$ are the dihedral angle and wing incidence angle, both at the wing root. The term $\eta$ denotes the Kelvin-Voigt damping coefficient. It need not be the same for bending and twisting in general. It is worth noting that the RHS of Eq. (1) depends on the rate of change of $\mathbf{u}_{B}$ which is obtained from the equations of motion of the complete aircraft. ${ }^{1}$ This couples the structural dynamics of the wing to the flight dynamics of the complete aircraft and makes control design a challenging assignment. Physically, $F_{\text {tip }}$ and $M_{\text {tip }}$ can be realized by using wing tip flaps, not unlike the outboard feathers on a bird's wings. Indeed, trailing edge effectors have been demonstrated to be effective at flutter suppression as well. ${ }^{16}$ On the other hand, $\delta_{R}$ and $\theta_{R}$ are easier to control and the capability for root actuation is present naturally in flapping wing aircraft.

For the sake of completeness, it is worth noting that the structural dynamic of the wing are coupled to the rigid flight dynamics primarily via aerodynamics and kinematics, and to a lesser extent via the resultant 
variation in mass distribution. The coupled equations of motion have the following structure:

$$
\begin{aligned}
& m\left(\dot{\mathbf{u}}_{B}+S\left(\boldsymbol{\omega}_{B}\right) \mathbf{u}_{B}\right)+\tilde{m} \int_{w}\left(\dot{\mathbf{u}}_{f}+S\left(\boldsymbol{\omega}_{B}\right) \mathbf{u}_{f}\right) d y=\mathbf{F}_{\text {net }} \\
& \left.J \dot{\boldsymbol{\omega}}_{B}+S\left(\boldsymbol{\omega}_{B}\right) J \boldsymbol{\omega}_{B}+\int_{w}\left(I_{p}(y) \dot{\boldsymbol{\omega}}_{f}+S\left(\boldsymbol{\omega}_{B}\right) I_{p}(y) \boldsymbol{\omega}_{f}\right)\right) d y=\mathbf{M}_{\text {net }}
\end{aligned}
$$

where: $\int_{w}$ denotes integration over the wing, and $\mathbf{F}_{\text {net }}$ and $\mathbf{M}_{\text {net }}$ represent the net external (aerodynamic + gravitational) force and moment on the aircraft. Furthermore, $\boldsymbol{\omega}_{B}$ and $\boldsymbol{\omega}_{f}=\left[\begin{array}{lll}0 & \dot{\theta} & 0\end{array}\right]^{\mathrm{T}}$ are vector representations of the aircraft angular velocity and the twist rate of the wing, with components in the aircraft body axes. The reader is referred to Ref. [1] for a detailed and more general derivation of the equations of motion.

\section{B. Problem Considered in this Paper}

Control methodology in this paper is developed systematically for a simple linear case with just the twist degree of freedom. Therefore, only the second equation in Eq. (1) is relevant to this paper. This is a considerable simplification, but it provides most of the necessary ingredients to design a controller for the complete system. The control objective is to ensure that

$$
\lim _{t \rightarrow \infty}\left(\int_{0}^{L} \theta(t, y) d y-H(t)\right)=0
$$

where $H(t)$ is a sufficiently smooth time-varying signal. Physically, the left hand side could be taken to command, for example, a desired value of the lift. In a linear setting, the net lift produced by the wing is given by $\int_{0}^{L} 0.5 \rho V^{2} c C_{l, \alpha} \theta$, where $\rho$ is the air density and $C_{l, \alpha}$ is the lift curve slope (measured per unit span of the wing). Depending on the aerodynamic theory, the left hand side of the above equation may be a nonlinear function of $\theta$. It is almost always a function of $y$, and a solution to this case is presented later in the paper. It may be possible to treat the aircraft speed and angular rates as disturbances while designing a controller for the wing structural dynamics, but this point needs further investigation.

\section{Boundary Control of Twisting Motion: Root Control}

Consider the case where the flexible wing needs to be controlled from the wing root, i.e., the PDE for wing twist is given by

$$
\begin{aligned}
& \theta_{t t}-b \theta_{t y y}-a \theta_{y y}=M \theta, \\
& \theta_{y}(L)=0, \quad \theta(0)=U(t) \quad \ldots \text { wing tip free, root displacement controlled }
\end{aligned}
$$

where $a=G J / I_{p}, b=\eta a$, and $M \theta=-x_{a} c F / I_{p}$ in Eq. (1). The net force, $F$, has been assumed to be linear in $\theta$. It is easy to check that the system has an infinite relative degree with respect to the output in Eq. (4). Thus, one needs to approach this problem in two steps. In the first step, the desired steady state response is determined. In the second step, the error dynamics between the system behavior and the desired steady state are stabilized using backstepping. ${ }^{9}$ Therefore, the control $U(t)$ may be decomposed into two parts

$$
U(t)=U_{d}(t)+u(t)
$$

where $U_{d}$ is the desired boundary condition at the wing root for which the integral objective, Eq. (4), is satisfied, while $u(t)$ is the stabilizing controller obtained from backstepping.

\section{A. Boundary Condition for the Desired Dynamics}

If the desired behavior is an equilibrium (i.e., $H(t) \equiv H$ ), then the steady state PDE is given by

$$
\theta_{d, y y}+\mu^{2} \theta_{d}=0, \quad \theta_{d, y}(L)=0, \theta_{d}(0)=U_{d}
$$

where $\mu^{2}=M / a$ and the subscript, $d$, denotes the desired (also, in this case, steady) state. The following control law, $U_{d}$, ensures that the condition Eq. (4) is satisfied.

$$
U_{d}=\frac{\mu H}{\tan (\mu L)}
$$


Alternatively, the desired behavior may be time varying in which case the boundary condition prescribed at the root needs to be determined separately. The boundary condition at the wing tip, on the other hand, is relatively easy to choose because, as shown in Sec. IV, the system has a relative degree of 2 for the output in Eq. (4) and the wing tip moment as the control input.

\section{B. Backstepping}

Backstepping is carried out in two steps: (a) Target dynamics are identified, and (b) a backstepping transformation converts the system dynamics (in this case, the error dynamics) into the target dynamics and the $u(t)$ is obtained in the process. The method described by Krstic and Smyshlyaev ${ }^{9}$ is used here. Let $z$ denote the error between the system state and the desired steady state value.

Next, consider the target dynamics given by

$$
\begin{aligned}
& v_{t t}-b v_{t y y}-a v_{y y}=(M-a p) v-b p v_{t}, \\
& v(0)=v_{y}(L)=0
\end{aligned}
$$

Using the method of separation of variables, it is easy to check that the eigenvalues of this system are the solutions of

$$
\begin{aligned}
& \lambda^{2}+b\left(\nu^{2}+p\right) \lambda+\left(a\left(\nu^{2}+p\right)-M\right)=0 \\
& \text { where } \nu=\frac{2 n+1}{2} \frac{\pi}{L}, \quad n=0,, 1,2, \ldots
\end{aligned}
$$

The target dynamics are stable if and only if the control design parameter, $p$, satisfies

$$
p>\frac{M}{a}-\frac{\pi^{2}}{4 L^{2}}
$$

A dummy spatial variable $x$ is introduced and the backstepping transformation between $z=\theta-\theta_{d}$ (the error between the actual dynamics and the desired steady state in Eq. (9)) and $v$ (the target dynamics for the error state) is given by

$$
v(t, y)=z(t, y)-\int_{L}^{y} k(y, x) z(t, x) d x
$$

It is helpful to recall that the $z$ dynamics are given by

$$
z_{t t}-b z_{t y y}-a z_{y y}=M z, \quad z(0)=u, \quad z_{y}(L)=0
$$

In order to solve for $k(x, y)$, substitute Eqs. (12) and (13) into Eq. (9). Next, isolating the coefficients of $v$ and $v_{t}$, the following Klein - Gordon PDE for $k(y, x)$ is derived ${ }^{9}$

$$
\begin{aligned}
& k_{x x}(y, x)-k_{y y}(y, x)=-p k(y, x) \\
& k(y, y)=\frac{p}{2}(L-y), \quad k_{x}(y, L)=0
\end{aligned}
$$

The control input is given by

$$
u(t)=z(t, 0)=-\int_{0}^{L} k(0, x) z(x) d x
$$

It remains to explain the derivation of $k(x, y)$. First, we derive expressions for $v_{t t}$ and $v_{y y}$.

$$
\begin{aligned}
& v_{t t}=z_{t t}-\int_{L}^{y} k(y, x) z_{t t}(x) d x \\
& =b z_{t y y}+a z_{y y}+M z-\int_{L}^{y} k(y, x)\left(b z_{t x x}+a z_{x x}+M z\right) d x \\
& =b z_{t y y}+a z_{y y}+M z-\int_{L}^{y} k(y, x) M z d x-\int_{L}^{y} k_{x x}(y, x)\left(b z_{t}(x)+a z(x)\right) d x \\
& -k(y, y)\left(b z_{t y}(y)+a z_{y}(y)\right)+k_{x}(y, y)\left(b z_{t}(y)+a z(y)\right)-k_{x}(y, L)\left(b z_{t}(L)+a z(L)\right)
\end{aligned}
$$


The expression for $v_{y y}$ can be derived as follows:

$$
\begin{aligned}
v_{y} & =z_{y}(y)-k(y, y) z(y)-\int_{L}^{y} k_{y}(y, x) z(x) d x \\
v_{y y} & =z_{y y}(y)-2 k_{y}(y, y) z(y)-k_{x}(y, y) z(y)-k(y, y) z_{y}(y)-\int_{L}^{y} k_{y y}(y, x) z(x) d x
\end{aligned}
$$

The expression for $v_{t y y}$ is similar to that for $v_{y y}$. Substitute Eqs. (16) and (17) into Eq. (9) and isolate the coefficients of $z, z_{y}$, and $z$ and $z_{y}$ in the integrand. This yields the PDE $k_{x x}-k_{y y}=-p k$ and one of the two boundary conditions, viz., $k_{x}(y, L)=0$. It also yields the condition $k(y, y)=\frac{p}{2} y+$ constant. From the first equation of (17), it follows that $k(L, L)=0$ since $z_{y}(L)=v_{y}(L)=0$. Therefore, $k(y, y)=\frac{p}{2}(L-y)$, which is the second boundary condition.

One can solve for $k(y, x)$ using successive approximations, as described in Ref. ${ }^{9}$ Alternately, under the transformation of coordinates $x \mapsto L-x$ and $y \mapsto L-y$, the BVP in Eq. (14) is identical to the one described by Krstic and Smyshlyaev. ${ }^{9}$ The solution is given in terms of the modified Bessel function $I_{1}$ :

$$
k(y, x)=-\frac{p}{2}(L-y) \frac{I_{1}\left(\sqrt{p\left((L-y)^{2}-(L-x)^{2}\right)}\right)}{\left.\sqrt{p\left((L-y)^{2}-(L-x)^{2}\right)}\right)}
$$

In summary, the control signal, $U(t)$, is given by Eqs. (6), (15) and (18).

\section{Discussion}

A few observations are worth noting here.

1. The procedure described in the previous subsection is called backstepping because the Volterra operator in Eq. (12) has a lower triangular structure similar to backstepping transforms for ordinary differential equations. It is a continuum analogue of the backstepping transformations in ODEs and allows the controller acting at the boundary to compensate for the undesired (unmatched) dynamics.

2. If the wing is reasonably stiff $\left(\frac{M I_{p}}{G J}=\frac{M}{a}<\frac{\pi^{2}}{4 L^{2}}\right)$, i.e., $G J>4 L^{2} M I_{p} / \pi^{2}$, the system can be stabilized with $p=0$, i.e., with no additional control input.

3. For stability, it is essential that $b>0$, and the Kelvin-Voigt damping coefficient is always positive. A negative damping could be introduced due to aerodynamics, but it can be compensated by the term $b p v_{t}$ and wing flutter can be prevented. The compensation in damping imposes an additional constraint on $p$.

4. The controller in Eq. (15) requires that the twist angle at all points on the wing be known. This difficulty can be circumvented by designing a PDE-based observer ${ }^{9}$ or, practically, using a series of distributed sensors and fitting their output with an a priori designed spline.

Recall the observation that for a "sufficiently" stiff wing, no additional stabilizing controller is required. As an illustration, consider the wing of a micro aerial vehicle (MAV). For such wings, $a \sim \mathcal{O}(E)>\mathcal{O}\left(10^{7}\right)$, where $E$ is the Young's modulus. Furthermore, $M \approx 0.06 V^{2} c^{2} / I_{p}$, where $I_{p} \approx 10^{-3} \times c^{4}$ is the second moment of area of the cross section whose thickness is assumed to be $1 \%$ of the chord length (a very conservative estimate). In such cases, $M \sim \mathcal{O}\left(5 \times 10^{5}\right)$. Therefore, $\frac{M}{a} \sim \mathcal{O}(0.05)$. On the other hand, with $L^{2} \approx 0.16$ (an aspect ratio of 8 , too generous for most MAV!), $\frac{\pi^{2}}{4 L^{2}} \approx 12$. Therefore, a typical MAV wing is stiff enough to be stable in the open loop. On the other hand, one would expect large wings flying at high speeds to experience loss of stability in the open loop - a phenomenon called wing divergence.

\section{Boundary Control of Twisting Motion: Wing Tip Control}

Although backstepping can be employed when the twisting moment at the wing tip is used as the control input, it turns out that there is a simpler alternative, as described in this section. It relies on the fact that the output has a finite relative degree with respect to the twisting moment. 


\section{A. Tip Boundary Control when All Parameters are Known}

As in the previous section, one can design a backstepping controller for the case where a control moment is applied to the free end $(y=L)$ of the wing while the other end $(y=0)$ is clamped. In fact, the procedure in both cases is identical, although the final expressions for the control law differ slightly. Alternately, insofar as MAV are concerned, one may do without a stabilizing controller. The "tracking half" of the controller $\left(U_{d}(t)\right.$ in Eq. (6)) may be designed using the output measurements. This method is useful for adaptive designs as well. We consider the wing model

$$
\theta_{t t}-b \theta_{t y y}-a \theta_{y y}=M \theta, \quad a>0,
$$

where the control input is a moment applied at the wing tip, $u(t)=\theta_{y}(L, t)$, and $b=\eta a=\eta G J / I_{p}$. The control objective could be assumed to be linear in $\theta$ because it is usually the total aerodynamic force or moment produced by the wing. It reduces to the form

$$
\int_{0}^{L} \theta(t, y) d y=H(t)
$$

The problem will be solved using a linear control approach. Let $e(t)=\int_{0}^{L} \theta(t, y) d y-H(t)$ denote the error which needs to be regulated. Then,

$$
\begin{aligned}
& \ddot{e}=\int_{0}^{L} \theta_{t t}(t, y) d y-\ddot{H}(t) \\
& =\int_{0}^{L}\left(a \theta_{y y}+b \theta_{t y y}+M \theta\right) d y-\ddot{H}(t) \\
& =a \theta_{y}(L)-a \theta_{y}(0)+b \theta_{t y}(L)-b \theta_{t y}(0)+M e(t)-\ddot{H}(t)+M H(t) \\
& =b \dot{u}(t)+a u(t)-a \theta_{y}(0)-b \theta_{t y}(0)+M e(t)-\ddot{H}(t)+M H(t)
\end{aligned}
$$

A dynamic controller of the form

$$
b \dot{u}(t)+a u(t)=\ddot{H}(t)-M H(t)-(M+k) e(t)-k_{c} \dot{e}(t)+a \theta_{y}(0)+b \theta_{t y}(0)
$$

renders the system into the spring-mass form

$$
\ddot{e}(t)+k_{c} \dot{e}(t)+k e(t)=0 .
$$

The control law in Eq. (22) is similar to internal model-based controllers and so much would be expected given that it does not rely on full state feedback. In fact, it suggests that $\theta$ need not be monitored or measured at all locations on the wing. Instead, only $\theta_{y}(0)$ needs to be measured or estimated. The reference signal $H(t)$ is known. It may be difficult to inject damping, $\dot{e}(t)$. This is because $\dot{e}(t)$ is the rate of change of the lift and in practice, would require differentiating noisy acceleration signals.

Another interesting observation is that although the PDE system had an infinite relative degree when the root twist was chosen as the control input, the relative degree is 2 when twisting moment at the wing tip is considered as the input. This facilitates the control law design in this section considerably. The control law design described is this section lends itself readily to adaptation should $a$ and/or $M$ be unknown. In the control law (22), $a$ and $M$ could be readily replaced by the corresponding estimated values obtained from a passive estimator.

Finally, if $\theta(0)$ as well as $\theta_{y}(L)$ can be controlled, it may be possible to ensure that the wing deformation produces the desired net lift as well as moment. This control scheme is physically realizable in MAVs. For example, flapping wing MAVs such as the robotic bat described in Ref. [17] has a twist angle actuator at the root. A small flap at the wing tip, not unlike the outboard feathers in a bird wing, can provide twisting moment at the tip.

\section{B. Adaptive Control}

Adaptive control is a useful method when system dynamics and/or parameters are unknown. The elastic properties of a linearly elastic structure can be characterized experimentally. The aerodynamic force and moment distribution would be usually hard to characterize or if characterized at all, would show a considerably 
nonlinear spatial distribution. For example, a simple rectangular wing has an elliptic lift distribution under nominal flight conditions. ${ }^{18}$ Nonlinearities would be considered in a later section of this paper. Consider the case where $M$ is constant, but unknown. This is not a very realistic model, but provides a sound foundation to design an adaptive controller.

Consider the dynamics in Eq. (19) with the objective in Eq. (20). The control law in (22) is modified so that

$$
b \dot{u}(t)+a u(t)=\ddot{H}(t)-\hat{M}(t)(H(t)+e(t))-k e(t)-k_{c} \dot{e}(t)+a \theta_{y}(0)+b \theta_{t y}(0)
$$

where $\hat{M}(t)$ is the estimated value of $M$. The error dynamics are given by

$$
\ddot{e}(t)+k_{c} \dot{e}(t)+k e(t)=-\tilde{M}(t)(H(t)+e(t))
$$

where $\tilde{M}(t)=\hat{M}(t)-M(t)$. An adaptive law must be designed for $\hat{M}(t)$ to ensure that the error, $e(t)$, goes to zero asymptotically.

For notational convenience, define $x=[e(t), \dot{e}(t)]^{\mathrm{T}}$ and $A=\left[\begin{array}{cc}0 & 1 \\ -k & -k_{c}\end{array}\right]$. Since $A$ is Hurwitz, it follows that there exists a positive definite symmetric matrix $P=P^{\mathrm{T}}>0$ satisfying the Lyapunov equation

$$
P A+A^{\mathrm{T}} P=-\mathbb{I}
$$

where $\mathbb{I}$ is the $2 \times 2$ identity matrix. We assume that $|M(t)|$ is bounded above by $B_{1}$ and $|\dot{M}(t)|$ by $B_{2}$, where $B_{1}$ and $B_{2}$ are constants.

Consider the Lyapunov function

$$
V(t)=x^{\mathrm{T}}(t) P x(t)+\frac{1}{\gamma} \tilde{M}(t)^{2}
$$

where $\gamma>0$ is the adaptive gain. Differentiating both sides with respect to $t$, we get

$$
\dot{V}(t)=-x^{\mathrm{T}}(t) x(t)+\frac{2}{\gamma} \tilde{M}(t)\left(\dot{\hat{M}}(t)-\gamma x^{\mathrm{T}} P\left[\begin{array}{l}
0 \\
1
\end{array}\right](e(t)+H)-\dot{M}(t)\right)
$$

With the adaptive law

$$
\dot{\hat{M}}(t)=\gamma \operatorname{Proj}\left(\hat{M}(t), x^{\mathrm{T}} P\left[\begin{array}{l}
0 \\
1
\end{array}\right](e(t)+H)\right)
$$

it follows that

$$
\dot{V}(t) \leq-x^{\mathrm{T}}(t) x(t)-\frac{2}{\gamma} \tilde{M}(t) \dot{M}(t) \leq \frac{-1}{\lambda_{\max }(P)}\left(x^{\mathrm{T}} P x+\frac{1}{\gamma} \tilde{M}(t)^{2}\right)+\frac{4 B_{1}^{2}}{\gamma \lambda_{\max }(P)}+\frac{4 B_{1} B_{2}}{\gamma}
$$

The projection law can be chosen so that $|\hat{M}(t)|<B_{1}$ (the same bound as that on $M(t)$ ). Therefore, it follows that

$$
\dot{V}(t) \leq \frac{-1}{\lambda_{\max }(P)}\left(1-\delta_{b}\right) V(t) \quad \forall V(t)>\frac{4 \lambda_{\max }(P) B_{1} B_{2}+4 B_{1}^{2}}{\delta_{b} \gamma}, \quad \delta_{b} \in(0,1)
$$

and hence, the solution is uniformly ultimately bounded with some $T \geq 0$ and with the ultimate bound given by

$$
\|x(t)\|_{\infty} \leq\|x(t)\|_{2} \leq \sqrt{\frac{4 \lambda_{\max }(P) B_{1} B_{2}+4 B_{1}^{2}}{\lambda_{\min }(P) \delta_{b} \gamma}} \forall t \geq T
$$

where $\delta_{b} \in(0,1)$. Note that the bound on $\|x(t)\|_{\infty}$ can be made arbitarily small by choosing a large $\gamma$. The steady state beam shape of the wing depends on the steady state value of the error $\tilde{M}(t)$. Finally, it is worth noting that although $a$ and $b$ were assumed to be known, the aforementioned analysis can be repeated to accommodate an unknown $a$ and $b$ as well. 


\section{Nonlinearities}

Two cases are presented in this section. The first case concerns a nonlinear twisting moment function. This problem is addressed via linearization. The second case considers a twisting moment which is linear in the twist angle, but with a spatially varying coefficient. The second problem is addressed using backstepping.

For brevity, only root actuation has been considered in this section. The analysis can be extended to actuation at the wing tip with minimal effort.

\section{A. Local Linear Analysis}

A nonlinear problem which is of interest to the present discussion would be to regulate a PDE of the form

$$
\theta_{t t}-b \theta_{t y y}-a \theta_{y y}=-x_{a} c F\left(\theta, \theta_{t}\right), \quad \theta_{y}(L)=0, \quad \theta(0)=U(t)
$$

where $x_{a}$ is the normalized (with respect to the chord length $c$ ) distance between the shear center and the aerodynamic center of the wing. The nonlinearity on the RHS arises from the inclusion of $\theta_{t}$ in the local flow velocity, and is widely used, for e.g., in flutter prediction. ${ }^{13}$ As in the earlier part of the paper, consider the error dynamics between the actual system and the steady state.

$$
\begin{aligned}
& \text { Desired: } \zeta_{t t}-b \zeta_{t y y}-a \zeta_{y y}=-x_{a} c F\left(\zeta, \zeta_{t}\right), \quad \zeta_{y}(L)=0, \zeta(0)=U_{d}(t) \\
& \text { Error Dynamics: } e_{t t}-b e_{t y y}-a e_{y y}=-x_{a} c F\left(\zeta, \zeta_{t}\right)-M\left(\zeta_{s s}, 0\right), \quad e_{y}(L)=0, e(0)=u(t)
\end{aligned}
$$

where the subscript 'ss' has been used to denote the steady state. Notice that, on this occasion, we have no simple expression for $U_{d}$. Instead, it must be calculated on a case-by-case basis for different nonlinearities on the right hand side. The nonlinear term can be approximated to the first order to get

$$
e_{t t}-b e_{t y y}-a e_{y y}=M_{2} e_{t}+M_{1} e, e_{y}(L)=0, e(0)=u(t)
$$

where the coefficients $M_{1}=-x_{a} c \frac{\partial F\left(\theta, \theta_{t}\right)}{\partial \theta}$ and $M_{2}=-x_{a} c \frac{\partial F\left(\theta, \theta_{t}\right)}{\partial \theta_{t}}$ depend on the steady state $\theta$.

Consider the following target dynamics for backstepping:

$$
\begin{aligned}
& v_{t t}-b v_{t y y}-a v_{y y}=M_{2} v_{t}+M_{1} v-a p v-b p v_{t}, \\
& v(0)=v_{y}(L)=0
\end{aligned}
$$

The characteristic equation is given by

$$
\lambda^{2}+\left(b \nu^{2}+b p-M_{2}\right) \lambda+\left(a\left(\nu^{2}+p\right)-M_{1}\right)=0
$$

where $\nu^{2}=\frac{\pi^{2}}{4 L^{2}}$. The target dynamics are stable if and only if

$$
p>\max \left\{\left(\frac{M_{1}}{a}-\nu^{2}\right),\left(\frac{M_{2}}{b}-\nu^{2}\right)\right\}
$$

The rest of the backstepping transformation is identical to that described in Section III-B.

\section{B. Lyapunov-based Analysis for Spatially-Varying $M(y)$}

In this section, we show that backstepping, described in Section III-B, can be used to control nonlinear systems of the form

$$
\theta_{t t}(t, y)-b \theta_{t y y}-a \theta_{y y}=M(y) \theta, \quad \theta_{y}(L)=0, \quad \theta(0)=U(t)=u(t)
$$

where $M(y)>0$ represents the moment distribution on the wing and is a function of the wing geometry. Physically, it captures the effect of downwash on a finite wing. ${ }^{18}$ There is no loss of generality, as one may infer from the analysis in Sec. III-B, in setting $U_{d}(t)=0$, which implies that $U(t)=u(t)$. The problem of tracking reduces to one of regulation. Stability analysis is completed in three steps: 
1. First, the backstepping transformation in Eqs. (12) and (14) is invoked to transform the system dynamics into "target" dynamics (see Eq. (40)).

2. The target dynamics are decomposed into "nominal" dynamics and a vanishing perturbation.

3. The nominal dynamics are shown to be exponentially stable, which implies that there exists a class of functions $M(y)$ for which the target system is exponentially stable.

The first step is to identify the target dynamics. Using the backstepping transformation (12), (14) and (18), i.e.,

$$
\begin{aligned}
& v(t, y)=\theta(t, y)-\int_{L}^{y} k(y, x) \theta(t, x) d x \\
& k(y, x)=-\frac{p}{2}(L-y) \frac{I_{1}\left(\sqrt{p\left((L-y)^{2}-(L-x)^{2}\right)}\right)}{\left.\sqrt{p\left((L-y)^{2}-(L-x)^{2}\right)}\right)},
\end{aligned}
$$

we obtain the target dynamics

$$
\begin{aligned}
v_{t t}(y)-b v_{t y y}-a v_{y y}-(M(y)-a p) v+b p v_{t} & =M(y) \int_{L}^{y} k(y, x) \theta(x) d x-\int_{L}^{y} k(y, x) M(x) \theta(x) d x, \\
& =\int_{L}^{y} M^{\prime}(x) \int_{L}^{x} k(y, z) \theta(z) d z d x \triangleq \Pi(x) \\
v(0)=v^{\prime}(L)=0, p>\max _{y} \frac{M(y)}{a} &
\end{aligned}
$$

The RHS of Eq. (40) may be viewed as a perturbation. The bound on the RHS is calculated as follows:

$$
\begin{aligned}
& \Pi(y) \leq\left(\max _{y} \int_{0}^{L}|k(y, z) \theta(z)| d z\right)\left(\int_{0}^{L}\left|M^{\prime}(x)\right| d x\right) \\
& \leq\left(\int_{0}^{L}\left|M^{\prime}(x)\right| d x\right)\left(\max _{y} \sqrt{\int_{0}^{L} k(y, z)^{2} d z}\right)\left(\sqrt{\int_{0}^{L} \theta(z)^{2} d z}\right)=K_{1}\|\theta\|
\end{aligned}
$$

where the constant $K_{1}$ is a (known) bound on $\left(\int_{0}^{L}\left|M^{\prime}(x)\right| d x\right)\left(\max _{y} \sqrt{\int_{0}^{L} k(y, z)^{2} d z}\right)$ and $\|\theta\|=\sqrt{\int_{0}^{L} \theta(z)^{2} d z}$ (the norm of $\theta$ ). Furthermore, as shown in Ref. [9], there exists a bounded function $l(x, y)$ such that

$$
\begin{aligned}
& \theta(t, y)=v(t, y)-\int_{L}^{y} l(y, x) v(t, x) d x, \\
& \Longrightarrow\|\theta\| \leq K_{2}\|v\|,
\end{aligned}
$$

where $K_{2}$ is another known constant. Hence, from Eqs. (41) and (42), it follows that

$$
\Pi(x) \leq K_{1} K_{2}\|v\|
$$

Hence, the perturbation on the RHS of Eq. (40) is vanishing in $v$.

Next, we prove the exponential stability of the nominal dynamics given by

$$
v_{t t}(y)-b v_{t y y}-a v_{y y}=(M(y)-a p) v-b p v_{t}, \quad v(0)=v^{\prime}(L)=0, p>\max _{y} \frac{M(y)}{a}
$$

In order to show stability, consider the Lyapunov function

$$
V(t)=\int_{0}^{L}\left(\frac{v_{t}^{2}}{2}+\frac{a v_{y}^{2}}{2}+(a p-M(y)) \frac{v^{2}}{2}+\delta v v_{t}\right) d y
$$


The above Lyapunov function is positive definite for a large enough $p>\max _{y} \frac{M(y)}{a}$ and small enough $\delta>0$. A bound for $\delta$ will be derived presently. Differentiating $V(t)$ with respect to $t$ yields

$$
\dot{V}(t)=\int_{0}^{L}\left(v_{t} v_{t t}+a v_{y} v_{t y}+(a p-M(y)) v v_{t}+\delta v_{t}^{2}+\delta v v_{t t}\right)
$$

The first term in the integrand can be expanded, followed by integration by parts, so that

$$
\begin{aligned}
& \int_{0}^{L} v_{t} v_{t t} d y=\int_{0}^{L}\left(b v_{t y y} v_{t}+a v_{y y} v_{t}-(a p-M(y)) v v_{t}-b p v_{t}^{2}\right) d y \\
& =\int_{0}^{L}\left(-b v_{t y}^{2}-a v_{y} v_{t y}-(a p-M(y)) v v_{t}-b p v_{t}^{2}\right) d y
\end{aligned}
$$

A similar expression can be calculated for $\int_{0}^{L} \delta v v_{t t} d y$. Substituting Eq. (47) into (46), it follows that

$$
\dot{V}(t)=\int_{0}^{L}\left((-b p+\delta) v_{t}^{2}-b v_{t y}^{2}-a \delta v_{y}^{2}-(a p-M(y)) \delta v^{2}\right) d y+\int_{0}^{L}\left(\delta b v v_{t y y}-\delta b p v v_{t}\right) d y
$$

Since $v(0)=v_{y}(L)=0$, it follows that

$$
\dot{V}(t)=\int_{0}^{L}\left((-b p+\delta) v_{t}^{2}-b v_{t y}^{2}-a \delta v_{y}^{2}-(a p-M(y)) \delta v^{2}\right) d y+\int_{0}^{L}\left(-\delta b v_{y} v_{t y}-\delta b p v v_{t}\right) d y
$$

Using Young's and Cauchy-Schwarz inequalities, it follows that

$$
\int_{0}^{L}\left(-\delta b v_{y} v_{t y}-\delta b p v v_{t}\right) d y \leq \frac{\delta b}{2} \int_{0}^{L}\left(\delta v_{y}^{2}+\frac{v_{t y}^{2}}{\delta}\right) d y+\frac{\delta b p}{2} \int_{0}^{L}\left(\delta v^{2}+\frac{v_{t}^{2}}{\delta}\right) d y
$$

Therefore,

$$
\dot{V}(t) \leq \int_{0}^{L}\left(\left(-b p+\delta+\frac{p b}{2}\right) v_{t}^{2}-\frac{b}{2} v_{t y}^{2}-(a-0.5 \delta b) \delta v_{y}^{2}-\left(a p-M(y)-\frac{\delta p b}{2}\right) \delta v^{2}\right) d y
$$

If $\delta$ is chosen such that

$$
\delta<\min \left\{1, \frac{(b-2 \kappa) p}{2}, \quad \frac{2(a p-M(y)-\kappa p)}{b p}\right\} \forall y \leq L
$$

where $\kappa$ is another small number such that $\kappa<\min (a-M(y) / p, b) \quad \forall y \leq L$, then it follows that

$$
\dot{V} \leq-\frac{\kappa \delta}{2} V
$$

This proves exponential stability and $\lim _{t \rightarrow \infty} v=0$. Note that the condition $p>\max _{y} \frac{M(y)}{a}+\kappa$ is stronger than the condition obtained for linear systems. Increasing $p$ allows us to choose a larger $\kappa$, subject to the constraint arising from $b$, to improve the guaranteed rate of convergence. Furthermore, since $\delta$ does not appear in the control law, it does not have any bearing on the performance and stability of the actual system.

The nominal system has been shown to be exponentially stable. Furthermore, the perturbation on the RHS of Eq. (40) is vanishing in $v$, as shown in Eq. (43). Hence, it follows that the nominal system is robust to sufficiently small perturbations. In particular, it is exponentially stable for sufficiently small $\int_{0}^{L}\left|M^{\prime}(y)\right| d y$ (e.g., see Lemma 9.1 in Khalil ${ }^{19}$ for an ODE analogue), i.e., for a sufficiently small $K_{1}$ in Eq. (41).

A theoretical bound on $K_{1}$ can be calculated by noting that, had we retained the perturbation term $\Pi(y) \triangleq \int_{L}^{y} M^{\prime}(x) \int_{L}^{x} k(y, z) \theta(z) d z d x$, it would have entered $\dot{V}(t)$ via terms involving $v_{t t}$ in Eq. (48). Using the Cauchy-Schwarz inequality, it can be shown that the change in $\dot{V}(t)$ arising from the inclusion of $\Pi(y)$ is given by

$$
\Delta \dot{V}(t) \leq 2 K_{1} K_{2} \underbrace{\left(1+\frac{\delta}{\sqrt{a p-M_{\max }}}\right)}_{K_{3}} V=K_{1} K_{2} K_{3} V
$$


Thus, if $K_{1}<\frac{\kappa \delta-2 \epsilon}{2 K_{2} K_{3}}$, where $2 \epsilon<\kappa \delta$, then the system continues to remain exponentially stable with a guaranteed convergence rate of $\epsilon$ for $V$.

Remark: Stability could have been proved even with $\delta=0$. In that case, $\dot{V}(t)=\int_{0}^{L}\left(-b p v_{t}^{2}-b v_{t y}^{2}\right) d y$ which is negative semi-definite. Clearly, the dynamics would converge to a steady state given by $a v_{y y}=(M(y)-a p) v$ and $v(0)=v_{y}(L)=0$. This BVP has the trivial solution $v \equiv 0$ as the only solution for almost all $M(y)$. The constant $p$ has to be chosen to prevent the existence of multiple solutions.

\section{Simulations}

Simulations are carried out in Matlab using a Galerkin-based approach to convert the PDE system into ODEs. The Galerkin truncation is not used as a basis for control law design, so no danger of a "spillover instability" arises. The twist $\theta(t, y)$ is expressed as a weighted sum of basis functions $\phi_{i}(y), i=1,2, \ldots, n$ and $\psi(y)$ :

$$
\begin{aligned}
& \theta(t, y)=s(t) \psi(y)+\sum_{1}^{n} \eta_{i}(t) \phi_{i}(y) \\
& \phi_{i}(0)=\phi_{i}^{\prime}(L)=0 ; \quad \psi(0)=0 ; \quad \psi^{\prime}(L)=1
\end{aligned}
$$

where $s(t)$ is the boundary control input for tip control. If boundary control has to be applied at the wing root, then $\psi(y)$ has to be chosen to satisfy $\psi^{\prime}(L)=0$ and $\psi(0)=1$.

The PDE in Eq. (19) can be rewritten as

$$
\begin{aligned}
& \psi(y) \ddot{s}(t)+\sum_{1}^{n} \ddot{\eta}_{i}(t) \phi_{i}(y)-\sum_{1}^{n} \phi_{i}^{\prime \prime}(y)\left(b \dot{\eta}_{i}(t)+a \eta_{i}(t)\right)-\psi^{\prime \prime}(y)(b \dot{s}+a s) \\
& =M\left(\psi(y) s+\sum_{1}^{n} \eta_{i}(t) \phi_{i}(y)\right)
\end{aligned}
$$

Using Galerkin's method, Eq. (54) is converted to a set of ODEs given by

$$
\mathbf{c}(\ddot{s}(t)-M s(t))+\mathbf{d}(b \dot{s}+a s(t))+[A](\ddot{\eta}(t)-M \eta(t))+[B](b \dot{\eta}(t)+a \eta(t))=0
$$

where

$$
\begin{aligned}
& \mathbf{c}=\int_{0}^{L} \psi(y) \phi(y), \quad \mathbf{d}=-\int_{0}^{L} \psi^{\prime \prime}(y) \phi(y), \\
& {[A]=\int_{0}^{L} \phi \phi^{T} d y, \quad[B]=-\int_{0}^{L} \phi\left(\phi^{\prime \prime}\right)^{T} d y}
\end{aligned}
$$

The reader will recall that $\psi$ is a scalar, and $\phi=\left[\begin{array}{ll}\phi_{1} & \phi_{2} \ldots \phi_{n}\end{array}\right]^{\mathrm{T}}$ is a vector. The control $s(t)$ is expressed similarly in terms of $\phi, \psi$ and $\eta$ to obtain a set of ODEs which are simulated to approximate the response of a twisting beam with boundary control. Figure 2 demonstrates the regulation of twist dynamics using the backstepping controller derived in Eq. (15), with the transformation in Eqs. (12) and (14). The value of $M / a$ was set to 8 , where $a=G J / I_{p}$. A value of $p=4$ yielded an unstable response, while the response was stable for $p=8$. Recall the following condition for stability with $L=1: p>M / a-\pi^{2} / 4 \approx 2.53$. The backstepping controller works even when $M(y)=M\left(1-y^{2}\right)$ is used (to mimic an elliptical lift distribution over the wing) instead of a constant $M(y) \equiv M$. The backstepping controller can be added on top of a tracking controller.

Figure 3 shows the simulation of a wing actuated by tip control. The value of $M / a$ was chosen so that stability is assured without the need for a dedicated stabilizing controller. The first plot was obtained for a controller designed assuming that all system parameters were known, whereas the right hand plot in the first row was obtained for a system where the aerodynamics were assumed to be linear but unknown. The third plot (bottom row) was obtained for the case where the aerodynamics were assumed to be affine in $\theta$, with an unknown spatially varying coefficient $M(y)$. In all cases, the twist amplitude converges to the steady state value with satifactory transients. The error metric Eq. (4) is also seen to be very small. 


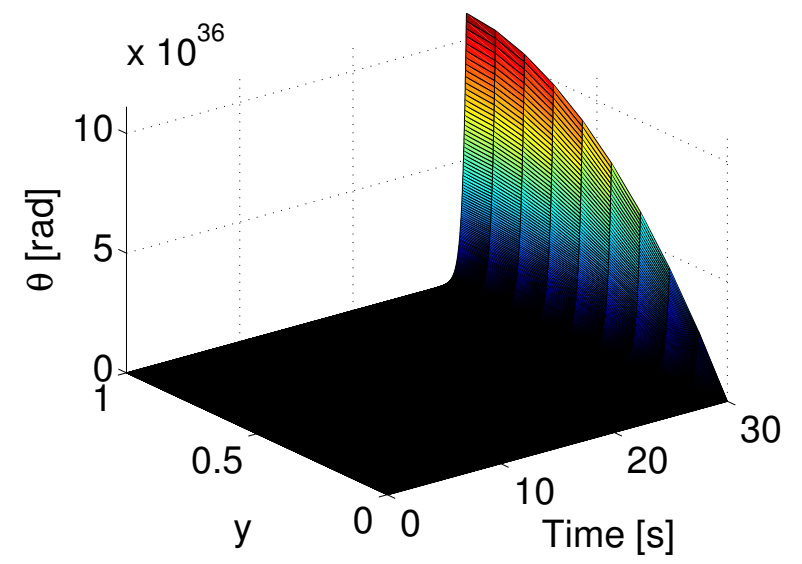

(a) Unstable response with $p=4$

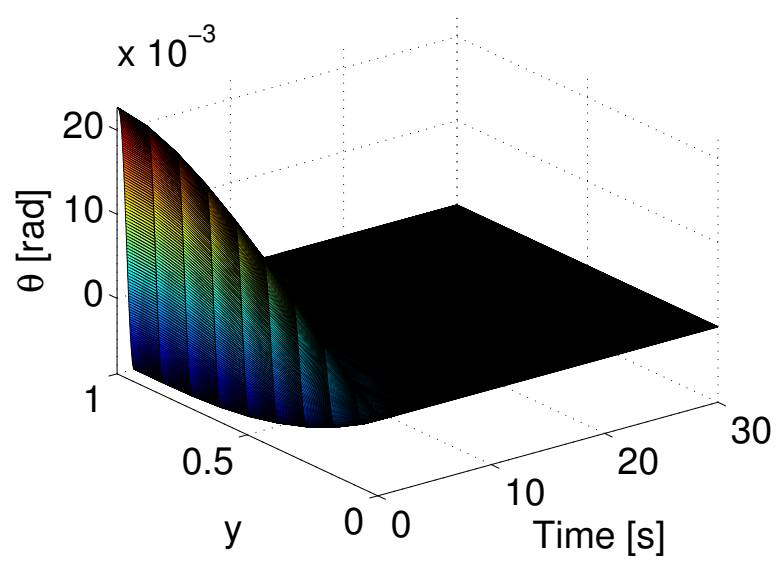

(b) Stable response with $p=8$

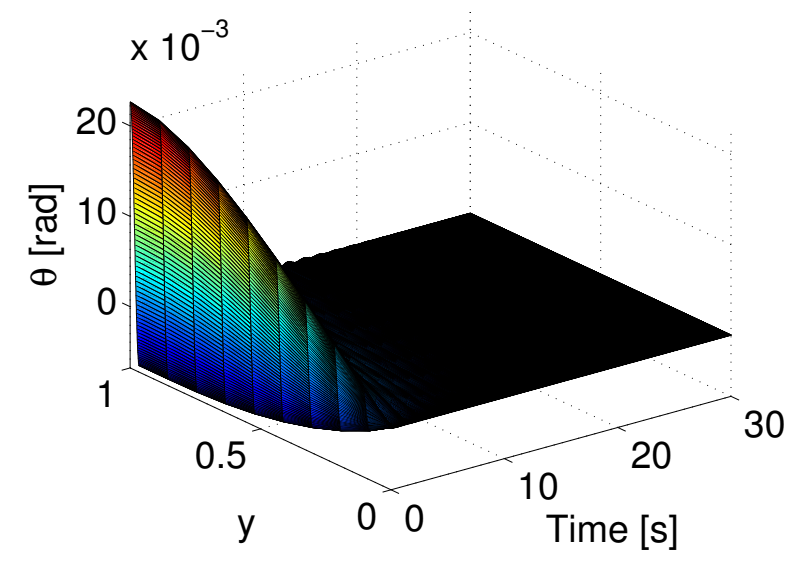

(c) Stable response with $p=8$ for spatially-varying $M(y)$

Figure 2. Regulation of the twist dynamics using the backstepping controller in Eq. (15), with the transformation in Eqs. (12) and (14). Both plots were obtained for $M / a=8$, while $p$ was increased to ensure stability. 


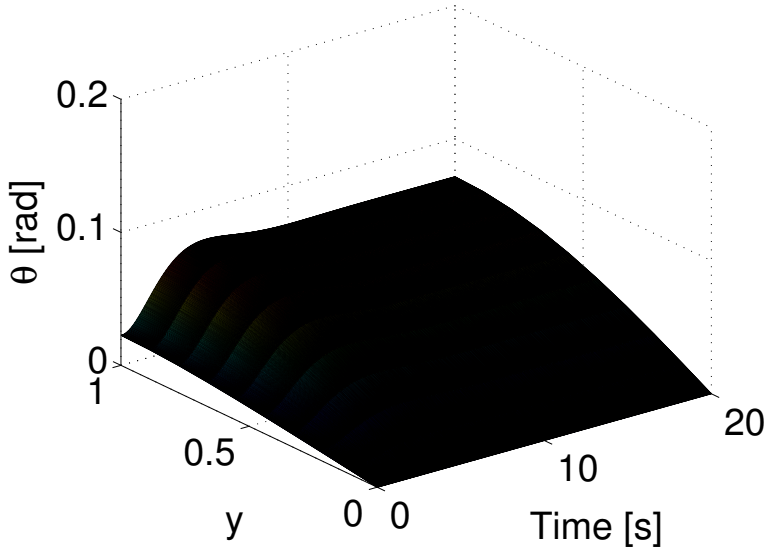

(a) All parameters known

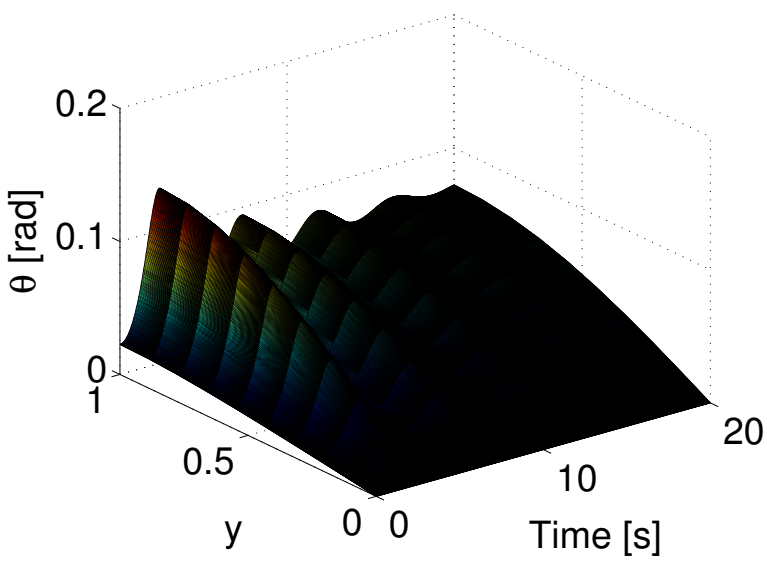

(c) Spatially varying, unknown $M(y)$

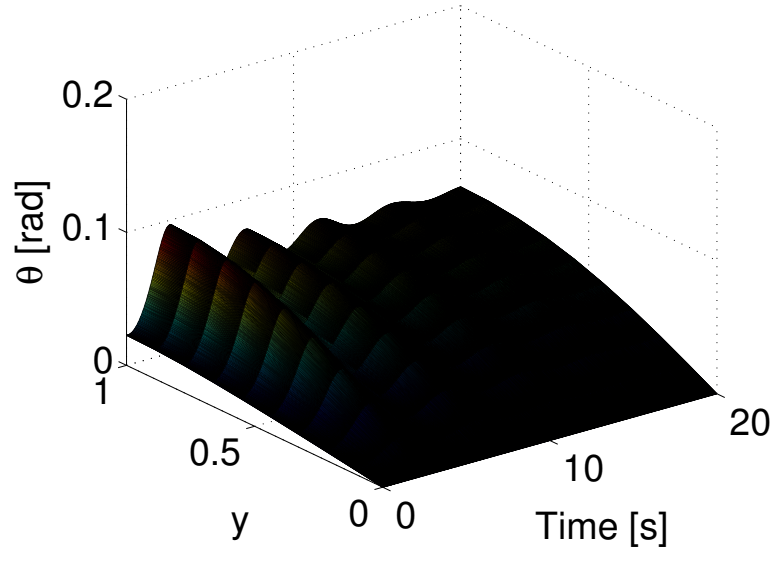

(b) Aerodynamics unknown

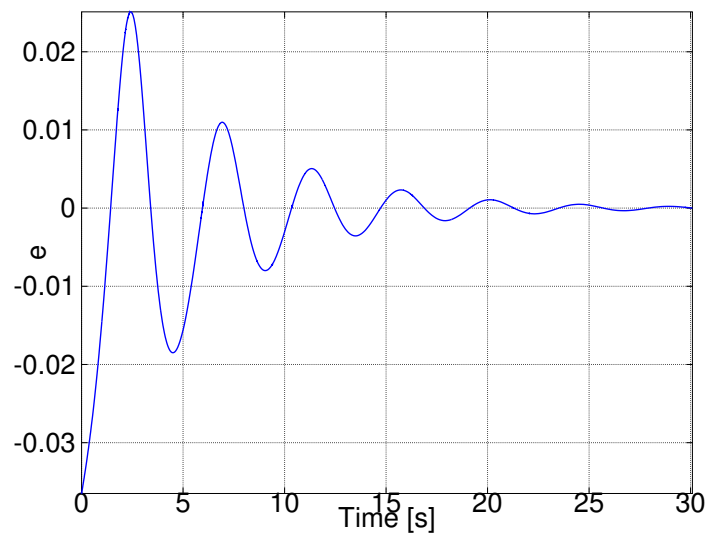

(d) Time history of $e(t)$

Figure 3. Twist profile of the wing as a function of time when the the adaptive controller in Eq. (24) is applied at the wing tip. Three cases have been considered here. In the first case, the system dynamics are assumed to be known. In the second case, the aerodynamics are assumed to be linear but unknown. In the third case, the aerodynamic moment is known to be of the form $M(y) \theta$, where $M(y)$ is spatially varying and unknown. The fourth plot shows the time history of $e(t)=\int_{0}^{1} \theta d y-H$, where $H=0.05$. 


\section{Conclusions}

This paper introduced a boundary control formulation for PDEs for the system output of a spatial integral of the state. This output function closely resembles the spatial distribution of an aerodynamic force, i.e., lift, that can be properly shaped as a function of time to control the rigid motions of the aircraft. In other words, the proposed boundary PDE control can significantly simplify the control of flexible aircraft by decoupling the force/torque control of the rigid body from the control of flexible wings to output the required force/torque input. In particular, the problem of beam twist was analysed in detail to illustrate the formulation. When the root twist angle was controlled, the output was seen to have an infinite relative degree with respect to the control input, and backstepping was employed for stabilization and tracking. Control laws based on this formulation ensure that the error between the reference output signal and the actual output decreases exponentially to zero when the system is fully known. When moment at the wing tip was controlled, it was seen that the output had a relative degree of 2 with respect to the control input. This facilitated the design of an adaptive controller along classical lines to ensure tracking. Both formulations were demonstrated by simulations. Future work would focus on two fronts: (a) extending the proposed approach to a wider class of nonlinearities, and (b) generalizing the method to the case of coupled bending and twist.

\section{Acknowledgment}

This project was supported in part by the Air Force Office of Scientific Research (AFOSR) under the Young Investigator Award Program (Grant No. FA95500910089) and the U.S. Army Research Office (ARO) under Award No W911NF-10-1-0296. The original problem of articulated wing aircraft was posed by Dr. Gregg Abate (AFRL).

\section{References}

\footnotetext{
${ }^{1}$ Paranjape, A. A., Chung, S.-J., Chakravarthy, A., and Hilton, H. H., "Dynamics and Performance of a Tailless MAV with Flexible Articulated Wings," AIAA Journal, in review.

${ }^{2}$ Paranjape, A. A., Chung, S.-J., and Selig, M. S., "Flight Mechanics of a Tailless Articulated Wing Aircraft," Bioinspiration ES Biomimetics, Vol. 6, No. 2, 2011.

${ }^{3}$ Tran, D. T., and Lind, R., "Parameterizing Stability Derivatives and Flight Dynamics with Wing Deformation," AIAA Atmospheric Flight Mechanics Conference 2010, AIAA Paper 2010-8227.

${ }^{4}$ Ifju, P.G., Jenkins, D. A., Ettinger, S., Yongsheng L., Shyy, W., and Waszak, M. R., "Flexible-wing-based micro air vehicles," AIAA $2002-0705$.

${ }^{5}$ Shyy, W., et al., "Recent Progress in Flapping Wing Aerodynamics and Aeroelasticity," Progress in Aerospace Sciences, Vol. 46, No. 7, 2010, pp.284-327.

${ }^{6}$ Abdulrahim, M., Garcia, H., and Lind, R., "Flight Characteristics of Shaping the Membrane Wing of a Micro Air Vehicle," Journal of Aircraft, Vol. 42, No. 1, 2005, pp.131 - 137.

${ }^{7}$ Russell D. L., "Controllability and Stabilizability Theory for Linear Partial Differential Equations: Recent Progress and Open Questions," Siam Review, Vol. 20, No. 4, 1978, pp. 639739.

${ }^{8}$ Krstic M., and Smyshlyaev A., "Adaptive Control of PDEs," Ann. Rev. in Control, Vol. 32, No. 2, 2008 , pp. 149 - 160.

${ }^{9}$ Krstic, M., and Smyshlyaev, A., Boundary Control of PDEs: A Course on Backstepping Designs, Ch. 7, pp. 79-88, Advances in Design and Control, SIAM, 2008.

${ }^{10}$ Christofides, P. D., and Daoutidis, P., "Feedback control of hyperbolic PDE systems," AIChE Journal, Vol. 42, No. 11, 1996, pp. 3063 - 3086.

${ }^{11}$ Christofides P. D., and Daoutidis, P., "Finite-Dimensional Control of Parabolic PDE Systems Using Approximate Inertial Manifolds," Proceedings of the 36th IEEE Conference on Decision and Control, 1997, pp. 1068 - 1073.

${ }^{12}$ Lohmiller, W., and Slotine, J.-J. E., "Contraction Analysis of Nonlinear Distributed Systems," International Journal of Control, Vol. 78, No. 9, 2005, pp. 678-688.

${ }^{13}$ Hodges, D. H., and Pierce, G. A., Introduction to Structural Dynamics and Aeroelasticity, Cambridge Aerospace Series (No. 15), Cambridge University Press, 2002, Ch. 2, pp. 59 - 66.

${ }^{14}$ De Queiroz, M., Dawson, D. M., Agarwal, M., and Zhang, F., "Adaptive Nonlinear Boundary Control of a Flexible Link Robotic Arm," IEEE Trans. Robotics and Automation, Vol. 15, No. 4, 1999, pp. 779 - 787.

${ }^{15}$ He, W., Ge, S. S., How, B. V. E., Choo, Y. S., and Hong, K.-S., "Robust Adaptive Boundary Control of a Flexible Marine Riser with Vessel Dynamics," Automatica, Vol. 47, 2011, pp. 722 - 732.

${ }^{16}$ Bieniawski, S., and Kroo, I. M., "Flutter Suppression Using Micro-Trailing Edge Effectors," $44^{\text {th }}$ AIAA/ASME/ASCE/AHS Structures, Structural Dynamics, and Materials Conference, AIAA 2003 - 1941.

${ }^{17}$ Chung, S.-J., and Dorothy, M., "Neurobiologically Inspired Control of Engineered Flapping Flight," Journal of Guidance, Control and Dynamics, Vol. 33, No. 2, 2010, pp. 440 - 453.

${ }^{18}$ McCormick, B. W., Aerodynamics, Aeronautics and Flight Mechanics, Wiley, 2nd Ed., 1994.

${ }^{19}$ Khalil, H. K., Nonlinear Systems, 3rd Ed., Pearson Education, Upper Saddle, NJ, 2000, pp. 340-345.
} 